\section{A cross-trial comparison of single-agent ibrutinib versus chlorambucil-obinutuzumab in previously untreated patients with chronic lymphocytic leukemia or small lymphocytic Iymphoma}

The treatment landscape for patients with chronic lymphocytic leukemia/small lymphocytic lymphoma (CLL/SLL) has evolved from single-agent/combination chemotherapies to anti-CD20 antibody-containing regimens, including chemoimmunotherapy, to novel agents targeting the B-cell receptor signaling pathway or BCL2. In older or less fit patients with comorbidities, standard first-line treatments include chlorambucil combined with anti-CD20 antibodies or single-agent ibrutinib. ${ }^{1}$ In the CLL11 study, chlorambucil-obinutuzumab showed superior efficacy compared with chlorambucil or chlorambucil-rituximab in patients with previously untreated CLL and comorbidities. ${ }^{2}$

Ibrutinib, a first-in-class, once-daily inhibitor of Bruton tyrosine kinase, is approved in the USA and Europe for the treatment of patients with CLL and allows for treatment without chemotherapy. In the ongoing RESONATE-2 phase III study (PCYC-1115/1116) in CLL/SLL, single-agent, first-line ibrutinib significantly improved progression-free survival (PFS), overall survival, and overall response rate compared with chlorambucil. ${ }^{3}$ Recently published results from the iLLUMINATE (PCYC-1130) phase III study showed superior PFS with first-line ibrutinib-obinutuzumab than with chlorambucil-obinutuzumab in patients with CLL/SLL, including patients with high-risk features $[\operatorname{del}(17 p) / T P 53$ mutation, del(11q), and/or unmutated IGHV].

Results of additional randomized studies evaluating single-agent ibrutinib versus standard chemoimmunotherapy regimens in first-line CLL were published recently: in the Alliance Intergroup (A041202) phase III trial, it was found that ibrutinib as a single agent or in combination with rituximab resulted in superior PFS compared with bendamustine-rituximab. ${ }^{5}$ However, to date, there are no data comparing single-agent ibrutinib with obinutuzumab-containing regimens. We performed a prespecified cross-trial analysis of the RESONATE- 2 and iLLUMINATE studies to compare outcomes with singleagent ibrutinib versus chlorambucil-obinutuzumab. Clinical trial registration: NCT01578707 and NCT02264574

This cross-trial analysis included all patients in the ibrutinib arm from RESONATE-2 and patients without $\operatorname{del}(17 p)$ from iLLUMINATE, given the exclusion of patients with del(17p) from RESONATE-2. Full details of the study design and eligibility criteria for both studies are described elsewhere ${ }^{3,4}$ and are summarized briefly in the Online Supplemental Methods.

The primary analysis was investigator-assessed PFS of patients treated with single-agent ibrutinib in RESONATE- 2 versus PFS of patients treated with chlorambucil-obinutuzumab in iLLUMINATE. Secondary analyses included investigator-assessed PFS in genomic high-risk patients [those with TP53 mutation, del(11q), and/or unmutated $I G H V]$, and medical resource utilization during the first 6 months on study treatment. The safety analysis included evaluation of adverse events collected for the time-matched analysis (first 6 months of study treatment) and for the entire follow-up.

An exploratory analysis was conducted for single-agent ibrutinib (RESONATE-2) versus ibrutinib-obinutuzumab (iLLUMINATE). This analysis comprised investigatorassessed overall response rate, including complete response; development of lymphocytosis [absolute lymphocyte count (ALC) increased $\geq 50 \%$ from baseline to $\left.\geq 5 \times 10^{\circ} / \mathrm{L}\right]$, duration and resolution of lymphocytosis (ALC decreased to baseline level or lower or $<5 \times 10^{9} / \mathrm{L}$ ); and time to normalization of ALC $\left(<4 \times 10^{\circ} / \mathrm{L}\right)$. Details of the statistical analysis are included in the Online Supplement.

Table 1. Patients' baseline demographics and disease characteristics.

\begin{tabular}{|c|c|c|}
\hline & $\begin{array}{l}\text { Thrutinib } \\
\mathrm{N}=136\end{array}$ & $\begin{array}{l}\text { Chlorambucil-obinutuzumab } \\
\qquad \mathrm{N}=98\end{array}$ \\
\hline \multicolumn{3}{|l|}{ Age, years } \\
\hline Median (range) & $73(65-89)$ & $73(40-86)$ \\
\hline$\geq 70, \mathrm{n}(\%)$ & $96(71)$ & $63(64)$ \\
\hline Time since initial diagnosis, months, median (range) & $30.5(1-241)$ & $42.9(0-480)$ \\
\hline Rai stage III/IV at screening, n (\%) & $68(50)$ & $49(50)$ \\
\hline \multicolumn{3}{|l|}{ ECOG performance status, $\mathrm{n}(\%)$} \\
\hline 0 & $61(45)$ & $44(45)$ \\
\hline $1-2$ & $75(55)$ & $54(55)$ \\
\hline Bulky disease (lymph node $\geq 5 \mathrm{~cm}), \mathrm{n}(\%)$ & $54(40)$ & $36(37)$ \\
\hline High-risk features (unmutated IGHV, del(11q), and/or TP53 mutation), n (\%) & $74 / 135(55)$ & $57(58)$ \\
\hline Unmutated $I G H V, \mathrm{n} / \mathrm{N}^{\mathrm{a}}(\%)$ & $58 / 98(59)$ & $45 / 90(50)$ \\
\hline $\operatorname{del}(11 q), n / N^{a}(\%)$ & $29 / 130(22)$ & 22/98 (22) \\
\hline TP53 mutation, $\mathrm{n} / \mathrm{N}^{\mathrm{a}}(\%)$ & $12 / 124(10)$ & $5 / 92(5)$ \\
\hline Any cytopenia, n (\%) & $72(53)$ & $51(52)$ \\
\hline Anemia (hemoglobin $\leq 11 \mathrm{~g} / \mathrm{dL}$ ) & $51(38)$ & $40(41)$ \\
\hline Thrombocytopenia (platelets $\leq 100 \times 10^{9} / \mathrm{L}$ ) & $35(26)$ & $21(21)$ \\
\hline Neutropenia (absolute neutrophil count $\leq 1.5 \times 10^{9} / \mathrm{L}$ ) & $10(7)$ & $1(1)$ \\
\hline
\end{tabular}

${ }^{N}$ : patients with available data. ECOG: Eastern Cooperative Oncology Group. 
A

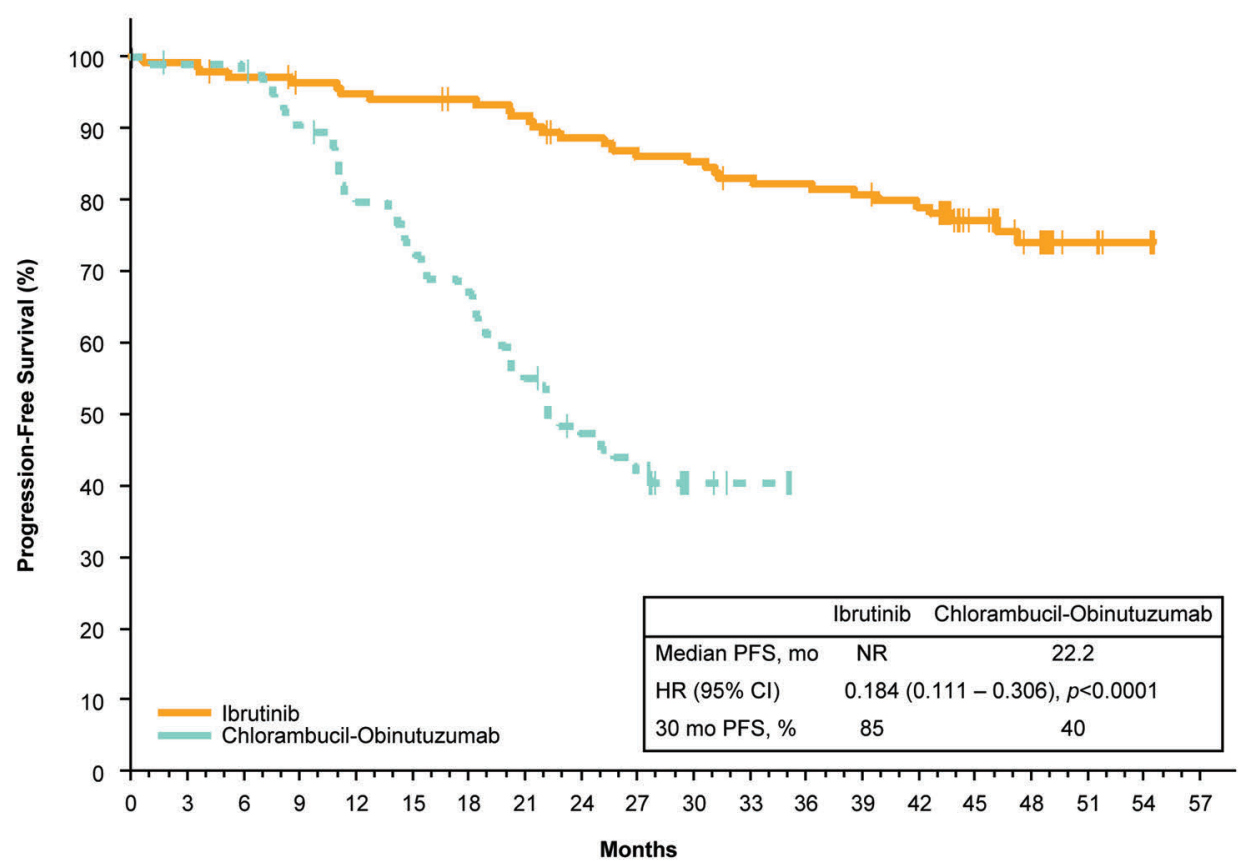

Patients at Risk

$\begin{array}{lrrrrrrrrrrrrrrrrrrr}\text { Ibrutinib } & 136 & 133 & 129 & 126 & 124 & 123 & 121 & 118 & 112 & 109 & 108 & 104 & 103 & 101 & 98 & 65 & 45 & 11 & 3 \\ \text { Chlorambucil-Obinutuzumab } & 98 & 94 & 93 & 85 & 74 & 68 & 63 & 51 & 42 & 37 & 7 & 5 & 0 & & & & & & \end{array}$

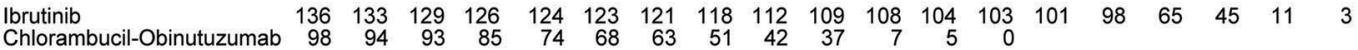

B

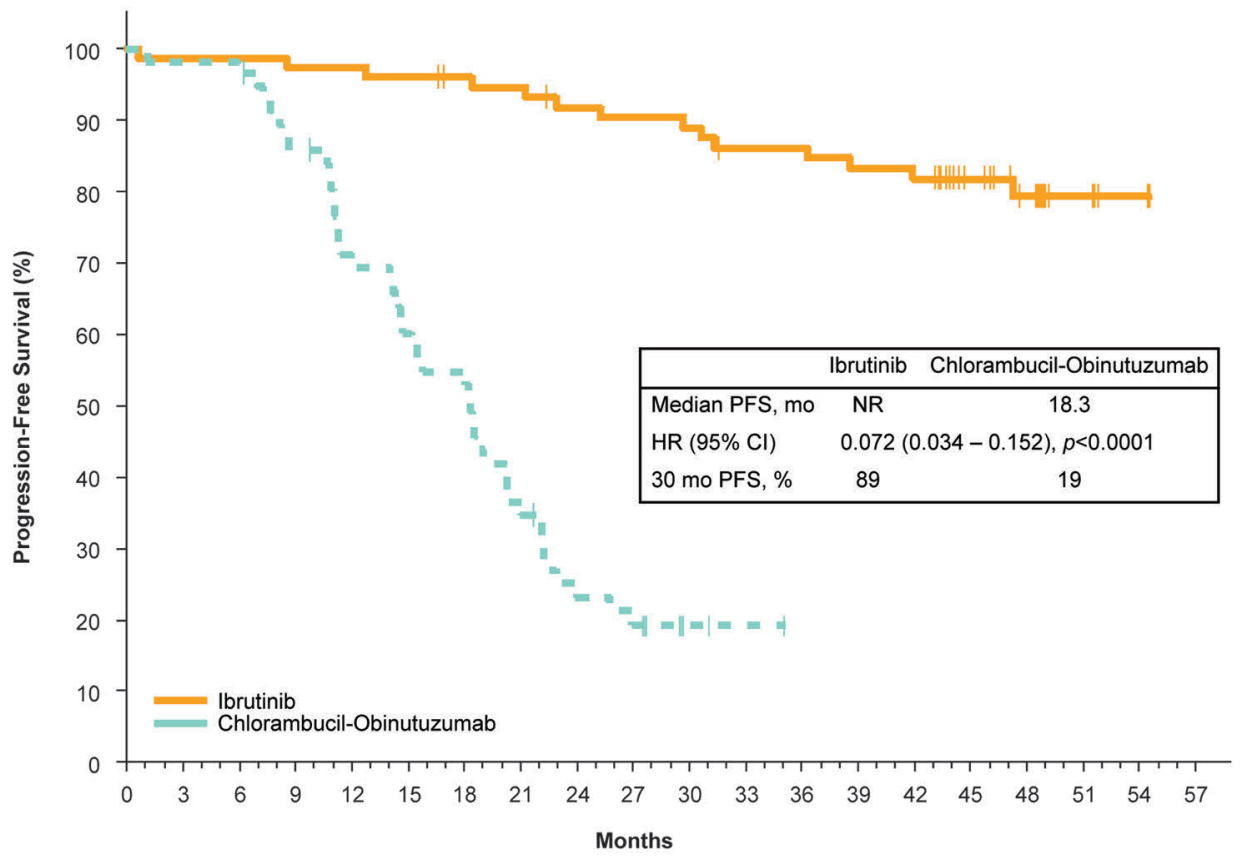

Patients at Risk

$\begin{array}{lrllllllllllllllllll}\text { Ibrutinib } & 74 & 73 & 73 & 72 & 72 & 71 & 69 & 68 & 65 & 64 & 63 & 60 & 60 & 58 & 57 & 43 & 30 & 9 & 2 \\ \text { Chlorambucil-Obinutuzumab } & 57 & 56 & 55 & 48 & 38 & 33 & 30 & 19 & 12 & 10 & 2 & 1 & 0 & & & & & & \end{array}$

Figure 1. Progression-free survival (investigator assessment) with single-agent ibrutinib versus chlorambucil-obinutuzumab. Kaplan-Meier curves of investigator-assessed progression-free survival are shown for (A) the overall population and (B) a high-risk population (patients with TP53 mutation, del(11q), and/or unmutated IGHV status). Vertical tick marks indicate patients with censored data. $95 \% \mathrm{Cl}$ : $95 \%$ confidence interval; NR: not reached; PFS: progression-free survival. 
Table 2. Summary of adverse events with single-agent ibrutinib and chlorambucil-obinutuzumab.

\begin{tabular}{|c|c|c|c|c|}
\hline & \multicolumn{2}{|c|}{$\begin{array}{l}\text { Ibrutinib } \\
\mathrm{N}=135\end{array}$} & \multicolumn{2}{|c|}{$\begin{array}{l}\text { Chlorambucil-obinutuzumab } \\
\qquad N=97\end{array}$} \\
\hline & AE reporting period ${ }^{\mathrm{a}}$ & First 6 months & AE reporting period ${ }^{\mathrm{a}}$ & First 6 months \\
\hline Median duration of treatment, months (range) & $46.9(0.7-54.5)$ & - & $5.1(0.0-6.3)$ & - \\
\hline Any grade $\geq 3 \mathrm{AE}, \mathrm{n}(\%)$ & $109(81)$ & $68(50)$ & $69(71)$ & $69(71)$ \\
\hline \multicolumn{5}{|l|}{ Most common non-hematologic grade $\geq 3 \mathrm{AE}, \mathrm{n}(\%)^{\mathrm{b}}$} \\
\hline Pneumonia & $16(12)$ & $4(3)$ & $4(4)$ & $3(3)$ \\
\hline Hypertension & $10(7)$ & $5(4)$ & $4(4)$ & $4(4)$ \\
\hline Hyponatremia & $7(5)$ & $2(1)$ & $1(1)$ & $1(1)$ \\
\hline Infusion-related reaction & 0 & 0 & $6(6)$ & $6(6)$ \\
\hline \multicolumn{5}{|l|}{ Hematologic grade $\geq 3 \mathrm{AE}, \mathrm{n}(\%)$} \\
\hline Neutropenia ${ }^{c}$ & $20(15)$ & $11(8)$ & $47(48)$ & $47(48)$ \\
\hline Anemia & $9(7)$ & $8(6)$ & $6(6)$ & $6(6)$ \\
\hline Thrombocytopenia $^{d}$ & $9(7)$ & $6(4)$ & $10(10)$ & $10(10)$ \\
\hline Febrile neutropenia & $5(4)$ & $1(1)$ & $7(7)$ & $7(7)$ \\
\hline AE leading to discontinuation of treatment, $n(\%)$ & $35(26)$ & $9(7)$ & $17(18)^{\mathrm{e}}$ & $17(18)^{\mathrm{e}}$ \\
\hline \multicolumn{5}{|l|}{ Most common AE leading to discontinuation, $\mathrm{n}(\%)^{\mathrm{t}}$} \\
\hline Infusion-related reaction & - & & $6(6)$ & \\
\hline Atrial fibrillation & $4(3)$ & & - & \\
\hline Neutropenia & - & & $4(4)$ & \\
\hline Chronic lymphocytic leukemia & $2(1)$ & & - & \\
\hline Death $^{g}$ & $2(1)$ & & - & \\
\hline Palpitations & $2(1)$ & & - & \\
\hline Pneumonia & $2(1)$ & 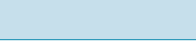 & - & \\
\hline
\end{tabular}

The median follow-up was 48.8 months for the ibrutinib arm of RESONATE- 2 and 31.3 months for both arms in iLLUMINATE for patients included in this cross-trial analysis. In total, 136 patients treated with single-agent ibrutinib and 98 patients treated with the chlorambucilobinutuzumab combination were included. The baseline characteristics of the patients were well balanced (Table 1). The median age of the patients was 73 years for both groups. The proportions of patients with high-risk genomic features were similar in the ibrutinib and chlorambucil-obinutuzumab groups $(55 \%$ and $58 \%$, respectively), as were the proportions with TP53 mutations $(10 \%$ and $5 \%)$, del(11q) $(22 \%$ and $22 \%)$, and/or unmutated IGHV (59\% and 50\%). Proportions with bulky disease $(\geq 5 \mathrm{~cm})$ were also comparable between groups (40\% and $37 \%$, respectively).

The PFS was significantly longer among patients treated with single-agent ibrutinib compared with those given chlorambucil-obinutuzumab (median not reached vs. 22.2 months), resulting in an $83 \%$ reduction in risk of progression or death with ibrutinib [hazard ratio (HR) $0.184 ; 95 \%$ confidence interval (95\% CI): 0.111-0.306]; $P<0.0001$ ] (Figure 1A). Results were similar when the HR was adjusted using a multivariate Cox regression model to account for differences in prognostic factors. The 30month PFS rates were $85 \%$ with ibrutinib and $40 \%$ with chlorambucil-obinutuzumab.

The magnitude of the PFS benefit with ibrutinib versus chlorambucil-obinutuzumab was even more pronounced among patients with high-risk features (median not reached $v s .18 .3$ months), with a $93 \%$ reduction in risk of progression or death (HR 0.072; 95\% CI: 0.034-0.152; $P<0.0001)$ (Figure 1B). Thirty-month PFS rates for highrisk patients were $89 \%$ versus $19 \%$, respectively. The PFS benefit with single-agent ibrutinib compared with chlorambucil-obinutuzumab was consistent across all subgroups examined, including patients with bulky disease (HR 0.063; 95\% CI: 0.024-0.164) (Online Supplementary Figure S1). For patients with unmutated IGHV [in this analysis, which excluded patients with $\operatorname{del}(17 p)]$, the PFS was longer among those treated with ibrutinib $(n=58)$ than among those treated with chlorambucil-obinutuzumab $(\mathrm{n}=45)$ (median not reached vs. 18.0 months; HR 0.074; 95\% CI:0.033-0.164; $P<0.0001)$ (Online Supplementary Figure S2). These results demonstrate sustained efficacy with single-agent ibrutinib with extended follow-up (median 4 years in RESONATE-2) regardless of high-risk genomic features. Given that the presence of $\operatorname{del}(11 q)$ or unmutated $I G H V$ is associated with poorer PFS outcomes among patients treated with standard chemoimmunotherapy, ${ }^{5-8}$ the updated International Working Group CLL guidelines recommend testing for these genomic abnormalities to aid in initial treatment decisions. ${ }^{9}$ Our results confirm prior observations that ibrutinib appears to diminish the negative prognostic impact of $\mathrm{del}(11 \mathrm{q})$ and unmutated IGHV observed with chemoimmunotherapy regimens. ${ }^{10,11}$ Additionally, these results, which used patient-level data, are consistent with a recent cross-trial study that reported favorable PFS with single-agent ibrutinib (from RES- 
ONATE-2) compared with published data from studies on first-line chemoimmunotherapy in CLL, ${ }^{12,13}$ particularly for patients with del(11q) or unmutated IGHV.

The median time to next treatment was not reached in either group (HR 0.115; 95\% CI: 0.055-0.242; $P<0.0001$ ). At the current follow-up, 11 patients $(8 \%)$ on singleagent ibrutinib and 39 patients $(40 \%)$ on chlorambucilobinutuzumab had received next-line treatment. The 30 month overall survival rates were $92 \%$ with ibrutinib and $84 \%$ with chlorambucil-obinutuzumab.

In a time-matched analysis, the rates of grade $\geq 3$ adverse events were lower with single-agent ibrutinib than with chlorambucil-obinutuzumab during the first 6 months of treatment (50\% and $71 \%$, respectively) (Table $2)$. The total adverse event reporting period was nine times longer for ibrutinib than for chlorambucil-obinutuzumab because of the longer treatment duration with ibrutinib [median 46.9 months (range, 0.7 to 54.5) and 5.1 months (range, 0 to 6.3), respectively]. During the total adverse event reporting period, grade $\geq 3$ adverse events occurred in $81 \%$ and $71 \%$ of ibrutinib- and chlorambucil-obinutuzumab-treated patients, respectively (Table 2 ). Rates of grade $\geq 3$ pneumonia ( $12 \%$ and $4 \%$ ), hypertension $(7 \%$ and $4 \%$ ), and hyponatremia $(5 \%$ and $1 \%$ ) were higher with ibrutinib than with chlorambucilobinutuzumab. As would be expected with chemoimmunotherapy regimens and consistent with recent reports, ${ }^{12,14}$ grade $\geq 3$ hematologic adverse events occurred at a higher rate with chlorambucil-obinutuzumab than with ibrutinib (Table 2), including neutropenia (48\% and $15 \%)$ and thrombocytopenia (10\% and $7 \%$ ), based on the total adverse event reporting period.

Adverse events leading to treatment discontinuation during the first 6 months occurred less frequently with ibrutinib compared with chlorambucil-obinutuzumab ( $7 \%$ and $18 \%$, respectively) (Table 2 ). During the total adverse event reporting period, adverse events leading to treatment discontinuations occurred in $26 \%$ and $18 \%$ of patients, respectively. The most common adverse events leading to treatment discontinuation were atrial fibrillation $(n=4)$ with ibrutinib, and infusion-related reaction $(n=6)$ and neutropenia $(n=4)$ with chlorambucil-obinutuzumab (Table 2). Overall, adverse events leading to treatment discontinuation were consistent with the known safety profiles of the individual drugs.

During the first 6 months of treatment, hospitalization rates were similar for patients treated with ibrutinib or with chlorambucil-obinutuzumab $(26 \%$ and $28 \%$, respectively) (Online Supplementary Table S1), while blood supportive products (16\% and $20 \%$ ) and growth factors ( $6 \%$ and $41 \%$ ) were administered less frequently with ibrutinib, reflecting the lower rate of grade $\geq 3$ neutropenia and thrombocytopenia seen with ibrutinib than with chlorambucil-obinutuzumab.

The exploratory analysis included 136 patients treated with single-agent ibrutinib and 99 patients treated with ibrutinib-obinutuzumab. The overall response rate (complete response + complete response with incomplete bone marrow recovery + nodular partial response + partial response) was similar between patients treated with ibrutinib or ibrutinib-obinutuzumab (91\% vs. $92 \%$, respectively) (Online Supplementary Figure S3). Although the complete response rate (including complete responses with incomplete bone marrow response) was higher for ibrutinib-obinutuzumab-treated patients than for ibrutinib-treated patients $(44 \%$ vs. $27 \%$, respectively; $P=0.006$ ), a long-term PFS benefit was achieved regardless of lower complete response rates with single-agent ibrutinib (Figure 1). A direct comparison of PFS outcomes between patients treated with ibrutinib or ibrutinib-obinutuzumab is limited because of the small number of patients who progressed on either regimen (median PFS not yet reached with both regimens) and differences in patient populations (Online Supplementary Table S2).

Lymphocytosis was observed in $57 \%$ of ibrutinib-treated and $8 \%$ of ibrutinib-obinutuzumab-treated patients (Online Supplementary Table S3). The median duration of lymphocytosis was 12.4 weeks (range, 0.1+ to 89.1+ weeks) with ibrutinib and 3.1 weeks (range, 1.3 to 19.0 weeks) with ibrutinib-obinutuzumab. As would be expected, attenuated lymphocytosis was observed with the addition of an anti-CD20 antibody to ibrutinib. Although lymphocytosis was more common with ibrutinib than with ibrutinib-obinutuzumab, resolution occurred in nearly all patients in both groups $(95 \%$ and $100 \%$, respectively). For patients with an elevated ALC at baseline, $80 \%$ of ibrutinib-treated and $98 \%$ of ibrutinibobinutuzumab-treated patients achieved normalization of the ALC (Online Supplementary Table S3) at a median time of 55.1 and 8.3 weeks, respectively.

Acknowledging the inherent limitations in a cross-trial comparison, results from this analysis suggest that outcomes are better in patients treated with single-agent ibrutinib than in those treated with chlorambucil-obinutuzumab, a standard regimen in first-line CLL, as demonstrated by the significant PFS benefit with ibrutinib. Because the present analysis excluded patients with $\operatorname{del}(17 p)$, these findings show superior PFS outcomes with ibrutinib than with chlorambucil-obinutuzumab even in the absence of del(17p). Importantly, the significant PFS benefit was also seen in patients with non$\operatorname{del}(17 p)$ high-risk genomic features [TP53 mutation, $\operatorname{del}(11 \mathrm{q})$, and/or unmutated IGHV] or bulky disease. In addition, in a time-matched analysis, the overall safety profile of single-agent ibrutinib appeared favorable compared to that of chlorambucil-obinutuzumab.

Alessandra Tedeschi, Richard Greil, ${ }^{2}$ Fatih Demirkan, ${ }^{3}$ Tadeusz Robak, ${ }^{4}$ Carol Moreno, ${ }^{5}$ Paul M. Barr, ${ }^{6}$

Bertrand Anz, 7 David Simpson, ${ }^{8}$ Gianluca Gaidano, ${ }^{9}$ Osnat Bairey, ${ }^{10}$ Don Stevens, ${ }^{11}$ Devinder Gill, ${ }^{12}$ Ian W. Flinn, ${ }^{13}$ Thomas J. Kipps, ${ }^{14}$ Jan A. Burger, ${ }^{15}$ Jennifer Lin, ${ }^{16}$

Thomas Webb, ${ }^{17}$ Viktor Fedorov, ${ }^{16}$ Lori Styles ${ }^{16}$ and John G. Gribben ${ }^{18}$

ASST Grande Ospedale Metropolitano Niguarda, Milan, Italy; ${ }^{2}$ Paracelsus Medical University Salzburg, Salzburg Cancer Research Institute, Cancer Cluster Salzburg, Salzburg, Austria; ${ }^{2}$ Dokuz Eylul University, Izmir, Turkey; ${ }^{4}$ Medical University of Lodz, Copernicus Memorial Hospital, Lodz, Poland; 'Hospital de la Santa Creu i Sant Pau, Autonomous University of Barcelona, Barcelona, Spain; ${ }^{6}$ Wilmot Cancer Institute, University of Rochester Medical Center, Rochester, NY, USA; ${ }^{7}$ Tennessee Oncology, Chattanooga, TN, USA; ${ }^{8}$ North Shore Hospital, Auckland, New Zealand; 'Amedeo Avogadro University of Eastern Piedmont, Novara, Italy; ${ }^{10}$ Rabin Medical Center, Beilinson Hospital and Sackler Faculty of Medicine, Tel Aviv University, Tel Aviv, Israel: "Norton Cancer Institute, Louisville, KY, USA; ${ }^{12}$ Princess Alexandra Hospital, Brisbane, Australia; ${ }^{13}$ Sarah Cannon Research Institute/Tennessee Oncology, Nashville, TN, USA, ${ }^{14}$ University of California, San Diego, Moores Cancer Center, La Jolla, CA, USA; ${ }^{15}$ The University of Texas MD Anderson Cancer Center, Houston, TX, USA; ${ }^{16}$ Pharmacyclics LLC, an AbbVie Company, Sunnyvale, CA, USA; '77anssen Ltd, High Wycombe, UK and ${ }^{18}$ Barts Cancer Institute, Queen Mary University of London, London, UK

Acknowledgments: the authors thank the patients who participated in this study, and their supportive families, as well as the investigators, sub-investigators, and coordinators at each of the study sites. 
Funding: this study was sponsored by Pharmacyclics LLC, an AbbVie Company. Editorial support was provided by Emily Chastain, $P h D$, an employee of Pharmacyclics LLC, an AbbVie Company.

Correspondence: ALESSANDRA TEDESCHI. alessandra.tedeschi@ospedaleniguarda.it doi:10.3324/haematol.2019.223743

Information on authorship, contributions, and financial \& other disclosures was provided by the authors and is available with the online version of this article at wWw. haematologica.org.

\section{References}

1. Eichhorst B, Robak T, Montserrat E, et al. Chronic lymphocytic leukaemia: ESMO clinical practice guidelines for diagnosis, treatment and follow-up. Ann Oncol. 2015;26(Suppl 5):v78-84.

2. Goede V, Fischer K, Busch R, et al. Obinutuzumab plus chlorambucil in patients with CLL and coexisting conditions. N Engl J Med. 2014; 370(12):1101-1110.

3. Burger JA, Tedeschi A, Barr PM, et al. Ibrutinib as initial therapy for patients with chronic lymphocytic leukemia. N Engl J Med. 2015;373(25):2425-2437.

4. Moreno C, Greil R, Demirkan F, et al. Ibrutinib plus obinutuzumab versus chlorambucil plus obinutuzumab in first-line treatment of chronic lymphocytic leukaemia (iLLUMINATE): a multicentre, randomised, open-label, phase 3 trial. Lancet Oncol. 2019;20(1):43-56.

5. Byrd JC, Gribben JG, Peterson BL, et al. Select high-risk genetic features predict earlier progression following chemoimmunotherapy with fludarabine and rituximab in chronic lymphocytic leukemia: justification for risk-adapted therapy. J Clin Oncol. 2006;24(3):437443.
6. Stilgenbauer S, Schnaiter A, Paschka P, et al. Gene mutations and treatment outcome in chronic lymphocytic leukemia: results from the CLL8 trial. Blood. 2014;123(21):3247-3254.

7. Thompson PA, Tam CS, O'Brien SM, et al. Fludarabine, cyclophosphamide, and rituximab treatment achieves long-term disease-free survival in IGHV-mutated chronic lymphocytic leukemia. Blood. 2016;127(3):303-309.

8. Eichhorst B, Fink AM, Bahlo J, et al. First-line chemoimmunotherapy with bendamustine and rituximab versus fludarabine, cyclophosphamide, and rituximab in patients with advanced chronic lymphocytic leukaemia (CLL10): an international, open-label, randomised, phase 3, non-inferiority trial. Lancet Oncol. 2016;17(7):928-942.

9. Hallek M, Cheson BD, Catovsky D, et al. iwCLL guidelines for diagnosis, indications for treatment, response assessment, and supportive management of CLL. Blood. 2018;131(25):2745-2760.

10. O'Brien S, Furman RR, Coutre S, et al. Single-agent ibrutinib in treatment-naive and relapsed/refractory chronic lymphocytic leukemia: a 5-year experience. Blood. 2018;131(17):1910-1919.

11. Kipps TJ, Fraser G, Coutre SE, et al. Integrated analysis: outcomes of ibrutinib-treated patients with chronic lymphocytic leukemia/small lymphocytic leukemia (CLL/SLL) with high-risk prognostic factors [abstract]. Hematol Oncol. 2017;35(Suppl 2):109-111.

12. Robak T, Burger JA, Tedeschi A, et al. Single-agent ibrutinib versus chemoimmunotherapy regimens for treatment-naive patients with chronic lymphocytic leukemia: a cross-trial comparison of phase 3 studies. Am J Hematol. 2018;93(11):1402-1410.

13. Van Sanden S, Baculea S, Diels J, Cote S. Comparative efficacy of ibrutinib versus obinutuzumab + chlorambucil in first-line treatment of chronic lymphocytic leukemia: a matching-adjusted indirect comparison. Adv Ther. 2017;34(7):1650-1661.

14. Woyach JA, Ruppert AS, Heerema NA, et al. Ibrutinib regimens versus chemoimmunotherapy in older patients with untreated CLL. N Engl J Med. 2018;379(26):2517-2528. 